\title{
NEFROPATÍA LÚPICA. CASO CLÍNICO Y REVISIÓN BIBLIOGRÁFICA
}

\author{
Nephropathy Lupus. Clinical Case and Literature review
}

Isis Judith Fajardo Hernández,* Sinia Coralia Andrade Sánchez,* Genis José Ramos Escalante,* Sherry Gabriela Melgar Manchamé,* Tania Soledad Licona Rivera.**

\section{RESUMEN}

El Lupus Eritematoso Sistémico (LES) es una enfermedad de carácter autoinmune sistémica, de etiología desconocida, sin embargo se estima que factores genéticos y ambientales contribuyen a la aparición de la enfermedad. Su incidencia es mayor en mujeres en edad reproductiva. Una de sus complicaciones es la Nefropatía Lúpica, la cual puede ser mortal para el paciente, por lo que se requiere un diagnóstico oportuno y tratamiento eficaz para mejorar su calidad de vida. Un ejemplo de dicha complicación es el caso clínico desarrollado en el presente artículo. Paciente femenina de 18 años de edad, con LES diagnosticada hace un año, ingresó a la sala de Nefrología del Hospital Mario Catarino Rivas (HMCR) con historia de 7 días de evolución de ascitis progresiva acompañado de dolor epigástrico y disnea progresiva. Laboratorialmente con: C3: $62.4 \mathrm{mg} / \mathrm{dl}$, C4: $5.0 \mathrm{mg} / \mathrm{dl}$, anticuerpos antinucleares (ANA): positivo, nitrógeno ureico en sangre (BUN) $91.6 \mathrm{mg} / \mathrm{dl}$, creatinina $5.5 \mathrm{mg} / \mathrm{dl}$. No se realizó biopsia renal por falta de insumos en el hospital. La paciente fallece a los 7 días de su hospitalización. Discusión Se puede sospechar nefritis en paciente que padece lupus, si hay hematuria u orina de apariencia espumosa, asimismo si sufre de hipertensión arterial o muestra signos de edema, características presentes en la paciente de nuestro caso clínico.

*Estudiantes de la carrera de Medicina de cuarto año. Escuela Universitaria de las Ciencias de la Salud EUCS. Universidad Nacional Autónoma de Honduras en el Valle de Sula.

** Profesor Titular, EUCS, UNAH-VS, Pediatra

Dirigir correspondencia a isisjudith@hotmail.com

Recibido: 8 de Agosto 2,014 Aprobado 15 de Octubre 2,014

\section{PALABRAS CLAVE}

Lupus Eritematoso Sistémico, Nefritis Lúpica, Sistema Inmune.

\section{ABSTRACT}

Lupus Erythematous Systemic (SLE) is a disease of autoimmune character systemic, of unknown etiology, however it is estimated that genetic factors and environmental contribute to the onset of the disease. Its incidence is higher in women of reproductive age. One of the complications is the kidney disease lupus, which can be fatal for the patient, so it requires a timely diagnosis and effective treatment to improve their quality of life. An example of this complication is the case clinically developed in the present article. Female patient of 18 year old with SLE diagnosed a year ago, joined the ward Mario Catarino Rivas (HMCR) with 7 days of evolution of progressive ascites accompanied by pain history epigastric and progressive dyspnea. Laboratorial: C3: $62.4 \mathrm{mg} / \mathrm{dl}$, C4: $5.0 \mathrm{mg} / \mathrm{dl}$, antinuclear antibody (ANA) positive, blood urea nitrogen (BUN) $91.6 \mathrm{mg} / \mathrm{dl}$, creatinine $5.5 \mathrm{mg} / \mathrm{dl}$. Not held renal biopsy due to lack of supplies in the hospital. The patient dies within 7 days of your hospitalization. Discussion may be suspected nephritis patient with lupus, if hematuria or urine foamy appearance, also if you have high blood pressure or shows signs of edema, features present in the patient in our case report.

\section{KEY WORDS}

Lupus Erythematous Systemic, Lupus Nephritis, Immune System. 


\section{INTRODUCCIÓN}

El Lupus Eritematoso Sistémico (LES) es una enfermedad crónica multisistémica de origen autoinmune, en la cual hay presencia de anticuerpos citotóxicos e inmunocomplejos circulantes que producen daño tisular, fallo orgánico y en ocasiones hasta la muerte. ${ }^{(1-4)}$ En el 2004, en Nueva York durante el VII Congreso Internacional del Lupus Sistemico se instituyo el 10 de Mayo como el día exclusivo para esta enfermedad, con el fin de dar a conocer cómo afecta a alrededor de 5 millones de personas en el mundo y de la que se diagnostican cada año más de 100 mil nuevos casos a nivel mundial. ${ }^{(5)}$

El LES es causado por interacciones entre ciertos genes de predisposición y factores ambientales, originando respuestas inmunitarias anormales. ${ }^{(4,6,7)}$ El resultado final de estas anormalidades es la producción sostenida de auto anticuerpos patógenos y la formación de complejos inmunitarios que se unen a ciertos tejidos provocando: secuestro y destrucción de células revestidas de IgG, liberación de factores de la quimiotaxis, fijación y segregación de proteínas que forman el complemento. $(1,6,8)$

Entre los factores ambientales relacionados con LES se mencionan: la exposición a la luz ultravioleta, sustancias químicas y fármacos como ser: procainamida, hidralazina, clorpromacina, isoniazida, propiltiouracilo, alfa metildopa, fenitoína, fenobarbital, aminas aromáticas, tricloroetileno, sílice y molibdeno ${ }^{(3,4,9)}$. También sustancias encontradas en las dietas como ser: alfalfa, psoralenos, fenilalanina y tirosina. ${ }^{(1,6,9)}$ Algunos estudios mencionan como desencadenantes de LES el tabaquismo y tintes para cabello (parafenilendiamina). ${ }^{(1,9)}$

Un $90 \%$ de los casos con LES corresponden a mujeres en edad reproductiva entre 16-55 años $^{(1,2,10)}$, con un porcentaje de $78 \%$ al $98 \%$ según las series de estudios realizados en comparación con los hombres. ${ }^{(1-6,11-13)}$ En un estudio realizado en las salas de Medicina Interna del Hospital Escuela Universitario en Tegucigalpa en el año 2006, se tomó una muestra de 100 casos con Lupus y demostró una mayor frecuencia en mujeres que en hombres con una relación 8:2.(2)

En el LES, el riñón sufre daño inflamatorio que ocasiona la pérdida de su función y puede conducir a Insuficiencia renal crónica terminal (IRCT), pero no todas las formas de afectación renal evolucionan indefectiblemente a la IRCT. $\left.{ }^{6}\right)$ Si bien es cierto que la evolución de la afección renal en el LES suele ser difícil de predecir, existen factores de tipo demográficos, clínicos, inmunológicos e histopatológicos que tratan de estimar el pronóstico de la nefropatía lúpica, ${ }^{(1)}$ que se estima afecta a más de la mitad de los pacientes con LES. ${ }^{(13)}$

Clasificación de la Nefritis Lúpica según la OMS (1995): ${ }^{(1,8,13)}$

Clase I: Glomérulos normales: Ningún hallazgo significativo (con todas las técnicas) como también resultado normal por histología, pero depósitos por Inmunofluorescencia ó Microscopía electrónica.

Clase II: Alteraciones mesangiales puras (mesangiopatía): Ensanchamiento mesangial y/o leve hipercelularidad (+) ó hipercelularidad moderada (++). 
Clase III: Glomerulonefritis focal y segmentaria: Lesiones necrotizantes activa, lesiones activas y esclerosantes y lesiones esclerosantes.

Clase IV: Glomerulonefritis difusa (proliferación mesangial severa, endocapilar o mesangiocapilar ylo extensos depósitos subendoteliales): Sin lesiones segmentarias, pero presenta lesiones necrotizantes activas, lesiones activas y esclerosantes y lesiones esclerosantes.

Clase V: Glomerulonefritis membranosa difusa: Glomerulonefritis membranosa pura, asociada con lesiones de clase II, III y IV.

\section{Clase VI: Glomerulonefritis esclerosante avanzada.}

Para el diagnostico de nefritis Lúpica primero se realiza un examen físico, el cual mostrará signos de disminución de la función renal con edema. ${ }^{(2,10)}$ La presión arterial puede estar elevada, se pueden escuchar sonidos anormales a nivel cardíaco y pulmonar. ${ }^{(10)}$

Los exámenes que se pueden llevar a cabo abarcan: Título de ANA, BUN y creatinina, niveles de complemento ( $C 3, C 4)$, análisis de orina y proteína en orina. La biopsia renal no se utiliza para diagnosticar la nefritis lúpica, sino para determinar cuál es el tratamiento adecuado. ${ }^{(3,10,13)}$

El objetivo del presente artículo, es exponer un caso de Nefropatía Lúpica, ya que en las salas de nuestros hospitales se está viendo una considerable incidencia de dicha complicación en pacientes con LES obligando al médico a enriquecer sus conocimientos $y$ habilidades, para poder diagnosticarlo tempranamente.

\section{CASO CLÍNICO}

Se presenta el caso clínico de paciente femenina de 18 años de edad, procedente de Tocoa, Colon, quien fue ingresada en la sala de nefrología del Hospital Mario Catarino Rivas (HMCR) con historia de 7 días de evolución de ascitis progresiva, acompañada de dolor epigástrico irradiado en forma de cinturón, tipo cólico, intensidad 10/10 en la escala análoga del dolor, exacerbado con la ingesta de alimentos y atenuado con acetaminofén (1g c/8 horas). Acompañado de disnea progresiva de moderados a pequeños esfuerzos, que se atenuaba con el reposo. Concomitantemente presentaba fiebre no cuantificada y tos de 1 día de evolución de expectoración verdosa, ortopnea la cual la obligaba a adquirir una posición en semifowler, utilizando 3 almohadas. Edema bilateral asimétrico de miembros inferiores más marcado en la pierna izquierda, sin cambio de coloración, frio, de consistencia blanda, sin predominio de horario, acompañado de mialgias.

Entre sus antecedentes patológicos de importancia: diagnosticada con LES e hipertensión arterial hace un año en dicho hospital; estuvo ingresada por un derrame pleural izquierdo, por lo cual ameritó toracotomía en el momento del diagnóstico de la enfermedad. Además en esa ocasión presentó síndrome convulsivo.

Laboratorialmente:

C3:62.4mg/dl, C4:5.0mg/dl, Anticuerpos Antinucleares (ANA): positivo; hemograma con leucocitosis y neutrofilia; creatinina 
creatinina: $5.2 \mathrm{mg} / \mathrm{dl}$ y potasio: 4.1 ; ultrasonido doppler: esteatosis hepática leve, engrosamiento de la pared vesicular, aumento de la ecogenicidad renal a nivel cortical en forma atenuada, sin alteración de la relación cortico medular ni tamaño, esplenomegalia, dilatación portal y ascitis.

El tratamiento farmacológico que recibió: ácido fólico 3mg al día; Prednisona 50mg 2 tabletas por día; Omeprazol $20 \mathrm{mg}$ cada 12 horas; Enalapril 20mg al día; Metoclopramida 1 ampolla intravenosa cada 8 horas; Vancomicina $1 \mathrm{~g}$ cada 8 horas y bicarbonato.

\section{DISCUSIÓN:}

El Lupus es una extraña afección autoinmunitaria, sistemática y crónica. Por lo general la edad de presentación es entre los 17 y 45 años, con una edad promedio de 30.5 años. Desconociéndose una explicación a este fenómeno, no obstante se cree que está relacionado con la disminución de la respuesta inmunológica que sucede en forma progresiva con la evolución de la enfermedad. ${ }^{(2)}$ Todo lo anterior coincide con nuestro caso clínico, ya que la paciente se encontraba en el rango de edad reproductiva, es del sexo femenino y raza mestiza. Otra de las literaturas consultadas nos asevera que el $90 \%$ de los pacietes corresponden a mujeres en edad reproductiva al momento del diagnóstico, aunque son susceptibles las personas de cualquier género, edad y grupo étnico; en conclusión la prevalencia más alta se encuentra en mujeres que en hombres. ${ }^{(6)}$ Cabe destacar que la manifestaciones clínicas de lupus en el género masculino son mucho más severas que en mujeres. En la infancia las niñas son afectadas 4,5 veces más frecuentemente que los niños. Los factores hormonales pueden explicar por qué el Lupus ocurre con mayor frecuencia en mujeres que en hombres y que sea más frecuente a partir de la adolescencia, cuando aumenta la producción de estrógenos en el organismo. Desde hace mucho tiempo a los reumatólogos les ha preocupado este hecho, mientras continúan las investigaciones no se puede decir que las hormonas provoquen Lupus, aún se desconoce la razón exacta de la mayor frecuencia en mujeres a partir de la adolescencia. ${ }^{(14)}$

Si bien es cierto que sus orígenes y causas son desconocidos, sin embargo se cree que existen factores que pueden influir en su desarrollo: ambientales o genéticos, ${ }^{(6,7)}$ pero en nuestro caso clínico, nada de lo anterior parece ser la etiología, porque la paciente no presenta antecedentes familiares de padecimiento de esta enfermedad, ni exposición a sustancias nocivas.

La enfermedad de Lupus se clasifica en diferentes tipos: Lupus eritematoso sistémico, discoide, cutáneo subagudo, neonatal y otro tipo que puede ser causado por medicamentos. ${ }^{(6)}$ El tipo de enfermedad de la paciente de nuestro estudio era Lupus Eritematoso Sistémico porque presentaba afectación de varios órganos manifestándose con: edema, hipertensión arterial, derrame pleural, disnea, dolor articular, ascitis, trombosis venosa profunda, pseudoalopecia y antecedentes de convulsiones. Además presentaba la mayor de las complicaciones médicas: diagnóstico de Nefritis lúpica aún sin determinar en el grado en que se encontraba. ${ }^{(7,11)}$

Se puede sospechar que un paciente padece nefritis lúpica o una glomerulopatía 
si la orina es sanguinolenta o de apariencia espumosa, asimismo si sufre de hipertensión arterial o muestra signos de edema en manos y pies, ${ }^{6,7)}$ características presentes en la paciente de nuestro caso clínico, a quien se le realizó un análisis de sangre en el cual reveló la presencia de ANA positivos, C3 y C4 disminuidos, Creatinina aumentada, BUN aumentado; todo esto generalmente se encuentra cuando la persona padece de esta enfermedad y junto a un examen de orina puede detectar si ha habido daño en el riñón, siendo esta una de las mayores complicaciones de L.E.S. ${ }^{(11)}$

También es necesario afirmar que la biopsia renal es la norma de oro para corroborar y dar seguimiento al diagnóstico de muchas enfermedades renales, logrando así un control de posibles recaídas, entre ellas tenemos la nefritis lúpica, porque solamente así se puede confirmar la gravedad de la enfermedad. Esta complicación se presenta cuando los auto anticuerpos del Lupus afectan las estructuras para el filtrado glomerular. Ese proceso anómalo conduce a inflamación renal y podría derivar en la presencia de hematuria, proteinuria, o alteración de la función renal e incluso insuficiencia renal y requerir diálisis o un trasplante de riñón. ${ }^{(7,11)}$ Desafortunadamente la paciente no tuvo la oportunidad de realizarse una biopsia renal, y debido a la falta de insumos en el hospital se hizo difícil la clasificación de la nefritis lúpica, sin embargo creemos que su estadio era avanzado porque falleció rápidamente.

Hoy en día se cuenta con tratamiento para la Nefritis Lúpica que consiste en corticosteroides e inmunosupresores. Nuestra paciente consumía en dosis elevadas corticosteroi- des, y esto se explica porque de acuerdo a la literatura aquellos pacientes con problemas graves o que suponen riesgo de vida, tales como inflamación en los riñones, compromiso pulmonar o cardíaco y síntomas relacionados con el sistema nervioso central, necesitan un tratamiento más "agresivo"; recientemente en los países desarrollados se ha utilizado mycofenolate para tratar esta enfermedad renal grave derivada del lupus. ${ }^{(11,14)}$ También se incluyen altas dosis de corticosteroides, como prednisona y medicamentos que suprimen el sistema inmunológico. Dentro de los inmunosupresores se incluye la azatioprina, ciclofosfamida y ciclosporina. Referente a la alimentación esta debe ser completa en cuanto a los nutrientes, y hasta la fecha no se ha logrado vincular ningún tipo de alimento a esta enfermedad. $(6,7,10,13)$

El pronóstico para una persona con esta enfermedad depende de la severidad de sus complicaciones. Según investigaciones, el desenlace clínico para personas con LES ha mejorado en años recientes, ya que muchos de los pacientes presentan síntomas leves. Las causas principales de muerte en el primer decenio de la enfermedad son actividad de la enfermedad sistémica, insuficiencia renal e infecciones; después, episodios tromboembólicos que se tornan en causas de mortalidad de frecuencia creciente. (6) La enfermedad tiende a ser más activa en los primeros años después del diagnóstico y en pacientes menores de 40 años.

La paciente de nuestro caso clínico falleció al año después de ser diagnosticada con Lupus Eritematoso Sistémico debido a complicaciones con la Nefritis Lúpica y además 
ya presentaba trombosis venosa profunda; dos de las principales causas de muerte en los primero años de la enfermedad.

Conclusión: Según los datos recopilados y el caso presentado se llega a la conclusión que la nefritis lúpica es una de las complicaciones más frecuentes que presentan los pacientes con lupus sistémico, siendo la complicación con índice de mortalidad elevada, y en nuestro país lamentablemente no se cuenta con el material médico necesario para su tratamiento e incluso hay carencia para realizar las pruebas diagnósti- cas preventivas, siendo un diagnóstico tardío en la enfermedad y complicando así la calidad de vida del paciente ó como el caso de nuestra paciente siendo el motivo de muerte.

\section{AGRADECIMIENTOS}

Al Dr. Manuel Amílcar Rocha Meza, médico internista con subespecialidad en nefrología, Jefe del servicio de nefrología del HMCR, por su asesoría y aportes valiosos brindados para la elaboración de este caso clínico.

\section{BIBLIOGRAFÍA}

1. Vallecillo MF, Becerra JE. Lupus Eritematoso Sistémico y Embarazo: Revisión de Literatura. Revista Médica Hondureña. 2006; 74:209-217. [Fecha de acceso mayo. 30, 2014) Disponible en: http://ns.bvs.hn/RMH/pdf/2006/pdf/Vol74-4-2006-9.pdf

2. Izaguirre Rodríguez E, Tovar Peña O. Caracterización sindrómica clínico laboratorial de pacientes con LES ingresadas en las salas de medicina interna del Hospital Escuela. Revista Médica de los Postgrados de Medicina UNAH. 2007; 10(2): 103-109. [Fecha de acceso junio. 12, 2014] Disponible en:

http://www.bvs.hn/RMP/pdf/2007/pdf/Vol10-2-2007-5.pdf

3. William J. Aaorginson. Lupus Eritematoso. Revista Médica Hondureña. 1960; 28(1). Sección B. [Fecha de acceso mayo. 30, 2014] Disponible en: http://www.bvs.hn/RMH/pdf/1960/pdf/Vol28-1-1960-2.pdf

Gómez-Puerta JA, Cervera R. Lupus 4. eritematoso sistémico. Medicina \& Laboratorio. 2008; 14:211-223. [Fecha de acceso junio 18, 2014] Disponible en: http://www.medigraphic.com/pdfs/medlab/myl-2008/myl085-6b.pdf

Conozca sobre Lupus. Suplementos.

5. Diario El Heraldo. 2 de mayo del 2014. [Fecha de acceso mayo. 30, 2014] Disponible en:

http://www.elheraldo.hn/csp/mediapool/sites/ElHeraldo/OtrasSecciones/ NuestrosSuplementos/story.csp? cid $=629676 \&$ sid $=1654 \&$ fid $=373$.

6. Bevra Hannahs Hahn. Lupus Eritematoso Sistémico. Harrison Principios de 
Medicina Interna. 18 ed. Editorial Mc Graw-Hill; 2012.

7. Abud-Mendoza C. Nefritis lúpica: avances en el conocimiento de su inmunopatogénesis sin los esperables logros terapéuticos. Reumatología Clínica. 2013; 9(2):77-79. [Fecha de acceso junio. 18, 2014]. Disponible en: http://apps.elsevier.es/watermark/ctl_servlet?_f=10\&pident_articulo=90193737 \&pident_usuario $=0 \&$ pcontactid $=\&$ pident_revis ta $=273 \&$ ty $=129 \&$ accion $=$ L\&origen= reuma\&web=http://www. reumatologiaclinica.org\&lan=es\&fichero= 273v09n02a90193737pdf001.pdf

8. Sánchez I, Sánchez V, Teruel C, Menéndez J. Nefritis Lúpica. Revista Española de Patología. 2002; 35(3). [Fecha de acceso junio. 19, 2014] Disponible en: http://www.patologia.es/volumen35/vol35-num3/35-3n04.htm

9. Marcano Marcel J. Monagas Andrea C. Rodríguez Anggie M. Ruotolo Antonio J. Terán José R. Urbaneja Diana C. Factores Asociados Con La Respuesta Inmunológica En El Lupus Cutáneo. Facultad de medicina, Universidad Central de Venezuela. [Fecha de acceso junio. 15, 2014] Disponible en: http://vitae.ucv.ve/pdfs/VITAE_2523.pdf

10. Nefritis Lúpica. Medline Plus. [sitio web] [Actualizado el 9/8/2013; [Fecha de acceso junio 19, 2014] Disponible en: http://www.nlm.nih.gov/medlineplus/spanish/ency/article/000481.htm

11. Avilés Izquierdo JA, Cano Martínez N,
Lázaro Ochaita P. Epidemiological Characteristics of Patients With Cutaneous Lupus Erythematosus. Actas Dermo-Sifiliográficas (English Edition). 2014; 105(1):69-73. [Fecha de acceso junio. 15, 2014] Disponible en:

http://www.sciencedirect.com/science/article/pii/S0001731013003414.

12. Velázquez-Cruz R, Jiménez-Morales $\mathrm{S}$, Ramírez-Bello J, Aguilar-Delfín I, Salas- Martínez G, Baca Ruíz V, et al. Lupus eritematoso sistémico (LES): genómica de la enfermedad. Gaceta Médica de México. 2012; 148:371-80. [Fecha de acceso junio 16, 2014] Disponible en: http://www.medigraphic.com/pdfs/gaceta/gm-2012/gm124e.pdf

13. Ruiz Irastorza G, Espinosa G, Frutos MA, Jiménez Alonso J, Praga M, Pallarés $\mathrm{L}$. Diagnóstico y tratamiento de la nefritis lúpica. Documento de consenso del Grupo de Enfermedades Autoinmunes Sistémicas (GEAS) de la Sociedad Española de Medicina Interna (SEMI) y de la Sociedad Española de Nefrología (S.E.N.). Nefrología. 2012; 32 Suppl (1):1-35. [Fecha de acceso junio. 15, 2014] Disponible en: http://www. revistanefrologia.com/revitas/P-E/P-E-SA11298.pdf

14. Frutos M, Praga M, Quereda C, Rivera F, Segarra A. Nefritis lúpica: en busca de un futuro mejor. Revista de Nefrología. 2012; 32(2):136-8. [Fecha de acceso junio. 12, 2014]. Disponible en: http:// www.revistanefrologia.com/revistas/P1-E534/P1-E534-S3440-A11374.pdf 